\title{
Filtering Joystick Data for Shooter Design Really Matters
}

\author{
Christoph Lürig ${ }^{1}$ and Nils Carstengerdes ${ }^{2}$ \\ ${ }^{1}$ Trier University of Applied Science \\ luerigafh-trier.de \\ ${ }^{2}$ German Aerospace Center \\ Nils. Carstengerdes@dlr.de
}

\begin{abstract}
Designing satisfactory, quick and precise control schemes for shooters on consoles remains one of the major game play programming challenges today. Besides the application of game situation specific control aids like soft locking even simple and game unspecific filtering approaches can improve the control quality significantly. In this paper we will objectify and quantify this effect that is well known among game developers as heuristic knowledge.
\end{abstract}

\section{Introduction and Related Work}

First and third person shooters play an important role on the current generation console market today. Those games are played with a controller consisting of two analogue sticks and follow the control scheme first introduced by the game Time Splitters on the PlayStation 2 [1]. In this scheme, the right analogue stick is used for aiming and looking and the left analogue stick is used for moving around the world. Especially the aiming function is more difficult to implement than on a PC shooter like Unreal [2], as the precision and range of an analogue stick is lower than a PC mouse.

Input schemes in general represent an important factor as they make up one third of the total interactivity cycle of listening, thinking and talking as explained by Crawford [6]. Input control schemes fall under the category of the listening aspect. It is well known to the experienced game developer that control schemes make up for a large part of the game play experience. Within the human computer interface community a series of publications for the analysis of pointing devices exist. MacKenzie et al. have analyzed aiming on several game related pointing devices $[3,4]$. The second paper includes an analysis on several variations of the Wiimote. These works used Fitt's task to measure aiming performance as also described in [5] and elaborate on the characteristics of the aiming device itself. Fitt's task requires aiming at a randomly selected marker out of a predefined set. In our measurement we generated target spots randomly with a predefined sum of distances.

In order to overcome the inherent difficulties of the analogue sticks of consoles basically two approaches exist today. One approach is to use contextual information to assist the gamer in aiming at objects. Frequently used is soft locking where the aiming point automatically gravitates towards a target, when it comes close to it. For instance this technique is used in the game Halo [7]. The variations of softlocking 
assists and their impact on player performance and game balancing were recently analyzed in the work of Bateman and Mandrick et al. [8].

Other practically applied approaches are context insensitive filtering approaches as described by Lürig [9]. In general, the subject of input data processing from joysticks got little attention in the academic scene yet. Exceptions are publications where joystick data is used to steer physical devices (see e.g. Chestnutt et al. [10]).

In this paper we focus on the analysis of the filtering approach and explain under which conditions and how it has advantages. As a basis of experiments a XBOX 360 controller was used. Besides always applying a simple and tight dead zone filter we compared (a) no filter, (b) a square type input filter, and (c) the combination of the square type filter with a damping filter and a ballistic filter.

The square type filter simply maps the input value to the sign attached square value. The dampening filter constitutes a low pass filter that is applied to the input. The ballistic filter has works as a high pass filter. Specifications of those filters are found in [9]. The square type filter and the damping filter were used by one of the authors in the game Splintercell Essentials and the PSP version of Ironman. Design and calibration of those filters are based on experience and experimentation.

Running through the complete analysis we deduced the following practical guidelines for a game where the user applies the right analogue stick for aiming or moving a reticule. These guidelines are also the hypotheses that are discussed in the result section.

1. The aiming velocity to move to the correct position is improved significantly by the square type filter and gets further improved by adding the damping filter.

2. The lower the frame rate is, the lower is the aiming velocity. Especially there is a significant drop in the step between 20 and 30 fps. The frame rate has no influence on filter performance. If one filter is better than another for one frame rate it remains the same for all frame rates.

3. Learning effects from repetitions exist. Inexperienced gamers exhibit larger learning effects than experienced ones. The advantage of the additional damping filter over the pure square type approach becomes significant with experienced gamers.

These conclusions are drawn from an experimental series that is based on automated logging of data while test participants are playing a game. This approach therefore falls into the category of applying quantitative methods in game design. The method is also referred to metric game design (see [11]). This is an approach that becomes increasingly popular in online game design and is also picked up by academia like in the publication of Zamitto et al. [12].

\section{Test Design and Procedure}

Measuring values on a control scheme in an actual game is difficult because in a real game many effects accumulate and causal dependencies can be hardly isolated. We opted for the implementation of a specific test bed that avoids those problems. Within the measurement program a test participant has to steer a reticule with the right analogue stick of a XBOX 360 controller over several markers shown on the screen. 
Participants were asked to complete a series of 64 aiming trials. Participants were not given any practice period prior to the experimental trials so they could not familiarize themselves with the experimental procedure. In each trial the participants saw a target marker and were asked to steer a reticule as fast as possible by using the right analogue stick of the XBOX controller. After reaching the marker, another marker appeared which the participant should reach as fast as possible. Each trial consisted of ten target markers visible one at a time.

A marker was considered reached when the reticule came to a complete halt within a radius of 10 pixels of the target. Target markers were generated randomly. In order to provide comparability, markers were always 100 to 300 pixels apart and the distance of two consecutive hops between markers was always 400 pixels. As a consequence, the total distance covered for 10 markers was always 2000 pixels. The screen resolution was set to $1024 * 768$ pixels. Several measurements were taken while a series of ten markers are completed.

This test bed was chosen because it reflected well the basic shooter mechanics of aiming in a very isolated and easy to measure way. In shooters the player also has to move a reticule over a defined target and then needs to press a fire button. Measurements were extracted during the experiment for a trial of 10 markers.

The study was a $4 * 4$ design. The predictor variables were filter technique (no filter, square type filter, damping filter and ballistic filter) and frame rate (60 fps, $30 \mathrm{fps}, 20$ fps and $15 \mathrm{fps}$ ). As a consequence the latency time between joystick input and shown frame were $16.67 \mathrm{~ms}, 33.33 \mathrm{~ms}, 50.0 \mathrm{~ms}$ and $66.66 \mathrm{~ms}$. All factors were within-subjects and there were four repetitions of each condition. The orderings of each condition were randomly assigned to each participant to compensate for possible learning effects across conditions. The criteria were total time to finish each trial (in seconds), proximity time (time within 50 pixels to the goal in each trial, measured in seconds), travelled distance with the reticule on the screen (in pixels) and travel velocity (calculated by travel distance/total time).

A series of 64 trials got automatically executed with each participant. The final results got written to three comma separated value files that can easily be imported into Excel for further analysis. Thirty volunteers participated in the study as part of study requirements in psychology at the University of Trier (Germany), with age ranging from 18 to 29 years, mean 21.6. Thirteen participants were male. Participants' game playing experience was also recorded, as it is possible that this is a confounding factor. Seventeen reported having experience with game consoles. The two statistical methods applied in this study are the and the Bonferroni-Holm t-Test procedure [13].

\section{Results}

To test our first hypothesis, we performed a 4 (filter) $* 4$ (frame rate) $* 4$ (repetition) repeated-measures ANOVA on the proximity times with game console experience as a between-subjects variable. We chose proximity time for our main analysis because we think that here the influence of filter techniques will be seen most clearly in the data. It will be recalled that we suppose that filter techniques improve control quality. In particular, the damping filter seemed most promising to us. A significant difference for filter technique $\left(\mathrm{F}_{3,81}=52.936, \mathrm{p}<.001\right)$ was found. Post-hoc, Bonferroni-Holm 
corrected (2-sided) t-tests revealed that the damping filter led to significantly shorter proximity completion times than the square type filter $\left(\mathrm{t}_{28}=2.93, \mathrm{p}=.007\right)$. The square type filter led to significantly shorter times compared to the no filter condition $\left(\mathrm{t}_{28}=5.19, \mathrm{p}<.001\right)$. The square type filter is even better than the ballistic filter $\left(\mathrm{t}_{28}=5.543, \mathrm{p}<.001\right)$. The ballistic filter is better than no filter $\left(\mathrm{t}_{28}=4.162, \mathrm{p}<.001\right)$. The ranking is therefore: Damping filter led to the shortest times, the square type filter was second, the ballistic filter third, and the worst was no filter. As the damping and the ballistic filter had the square type filter as a preprocessing step, the additional component in the ballistic filter itself made things worse. This is no surprise as the ballistic filter has the opposite impact of the damping filter.

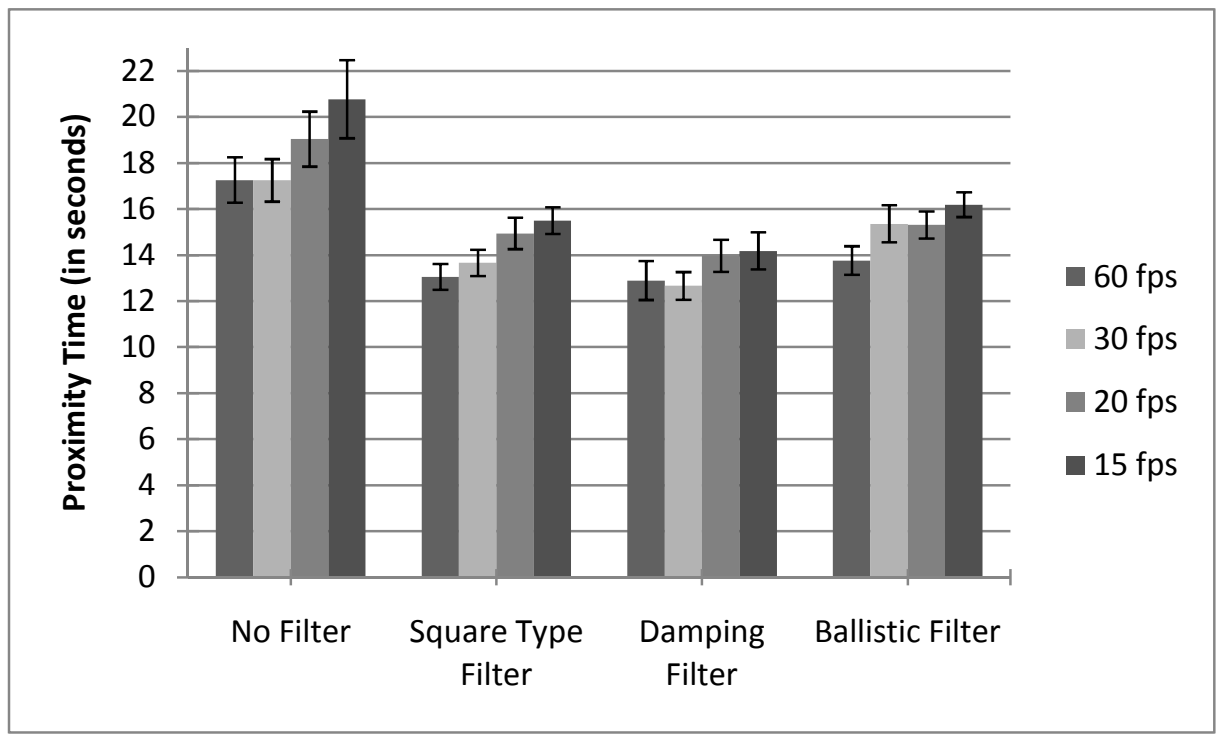

Fig. 1. Proximity times by filter technique and frame rate. Error bars show standard error

Our second hypothesis concerned the influence of frame rate on control quality. Our primary analysis was here the aforementioned $4 * 4 * 4$ repeated-measures ANOVA on proximity times. A higher frame rate should lead to shorter proximity times. The analysis revealed highly significant differences in proximity times regarding frame rate $\left(\mathrm{F}_{3,81}=18.304, \mathrm{p}<.001\right)$. Post-hoc, Bonferroni-Holm corrected (2-sided) t-tests revealed that proximity times for the highest frame rate $(60 \mathrm{fps})$ were slightly shorter than for the condition with $30 \mathrm{fps}$, but this difference did not reach statistical significance $\left(\mathrm{t}_{28}=1.971, \mathrm{p}=.059\right)$. The proximity times in the $20 \mathrm{fps}$ frame rate condition were significantly longer than in the $30 \mathrm{fps}$ frame rate condition $\left(\mathrm{t}_{28}=2.849\right.$, $\mathrm{p}=.008$ ). Therefore, a higher frame rate led to shorter times in our aiming task. This effect was most pronounced between the frame rates of $30 \mathrm{fps}$ and $20 \mathrm{fps}$. The latter (and even more the frame rate of $15 \mathrm{fps}$ ) should be avoided to improve control quality.

Interestingly, the frame rate had no influence on the filter performance: the interaction of frame rate and filter did not reach statistical significance $\left(\mathrm{F}_{9,243}=1.279\right.$, 
$\mathrm{p}=.249)$. In another $4 * 4 * 4$ repeated-measures ANOVA we analyzed whether frame rate had an effect on travelled distance. This is not the case $\left(\mathrm{F}_{3,84}<1\right)$.

Our third hypothesis is concerned with learning effects from repetitions. To verify this assumption we computed the $4 * 4 * 4$ repeated-measures ANOVA on proximity times mentioned above. A significant influence of repetition was found $\left(\mathrm{F}_{3,81}=17.36\right.$, $\mathrm{p}<.001)$. Post-hoc, Bonferroni-Holm corrected (2-sided) t-tests revealed shorter proximity completion times with every repetition (Trial 1 to Trial $2: \mathrm{t}_{28}=2.584$, $\mathrm{p}=.015$; Trial 2 to Trial 3: $\mathrm{t}_{28}=4.249, \mathrm{p}<.001$; Trial 3 to Trial $4: \mathrm{t}_{28}=2.832, \mathrm{p}=.008$ ). As stated in the introduction, we found larger learning effects for inexperienced gamers. The interaction of repetition and game console experience was significant $\left(\mathrm{F}_{3,81}=6.214, \mathrm{p}=.001\right)$. For inexperienced gamers the difference between Trial 1 and 2 was not significant $\left(\mathrm{t}_{11}=2.128, \mathrm{p}=.057\right)$ whereas the differences between Trial 2 and 3 $\left(\mathrm{t}_{11}=3.377, \mathrm{p}=.006\right)$ and Trial 3 and $4\left(\mathrm{t}_{11}=3.95, \mathrm{p}=.002\right)$ were significant. The inexperienced gamers got faster with every repetition. Experienced gamers showed a significant improvement from Trial 1 to $2(\mathrm{t} 16=2.503, \mathrm{p}=.024)$ and from Trial 2 to 3 $\left(\mathrm{t}_{16}=2.997, \mathrm{p}=.009\right)$ but not from Trial 3 to $4\left(\mathrm{t}_{16}<1\right)$. Experienced gamers did not get faster after the third trial.

Furthermore, we took a closer look at the interaction of filter and game console experience, which was also significant $\left(\mathrm{F}_{3,81}=13.808, \mathrm{p}<.001\right)$. This interaction could also be interpreted as a learning effect. Maybe experienced gamers need other filter techniques or take better advantage of certain kind of filters. In post-hoc, BonferroniHolm corrected (2-sided) t-tests the difference between the square type filter and the damping filter was significant for the experienced gamers $\left(\mathrm{t}_{16}=6.503, \mathrm{p}<.001\right)$ but not for the inexperienced gamers $\left(t_{11}<1\right)$. Thus, the proximity times were significantly shorter using the damping filter, if the user has game console experience. For inexperienced gamers in our experiment, using the square type filter did not lead to significantly longer proximity times than using the damping filter.

\section{Conclusion}

In this paper we showed that applying filters for analogue joysticks on joypads may significantly improve aiming velocity in shooter type playing scenarios. Several filtering types and their consequences were discussed. Of the discussed filters the damping filter that contains the square type filter as a preprocessing step was the best. This is consistent with the observation that the ballistic filter is worse than the square type filter, as the impact of the ballistic component is the opposite of the damping component.

\section{References}

1. Time Splitters: Free Radical Design, published by Eidos Interactive, PlayStation 2 (2000)

2. Unreal: Epic Games, published by GT Interactive, PC (May 22, 1998)

3. Natapov, D., MacKenzie, I.: The trackball controller improving the analog stick. In: Proceeding of the 2010 Conference on Future Play, pp. 199-206 (2010)

4. McArthur, V., Castelucci, S., MacKenzie, I.: An Empirical Comparison of "Wiimote" Gun Attachments for Pointing Tasks. In: EICS 2009, pp. 203-208. ACM, New York (2009) 
5. Oh, J., Stuerzlinger, W.: Laser Pointer as Collaborative Pointing Devices. In: Graphics Interface 2002, pp. 141-149 (2002)

6. Crawford, C.: The art of interactive design: A Euphonious and Illuminating Guide to building successful software. No Starch Press (2002)

7. Halo: Bungie Studios, published by Microsoft Game Studios, XBOX 2002 (2002)

8. Bateman, S., Mandryk, R., Statch, T., Gutwin, C.: Target Assistance for Subtly Balancing Competetive Play. In: CHI 2011 (2011)

9. Lürig: Filtering of analogue sticks on joypads for improved control precision. In: ACM FuturePlay 2007, pp. 197-200 (2007)

10. Chestnutt, et al.: An intelligent joystick for biped control. In: IEEE International Conference on Robotics and Automation (2006)

11. Hecker, C.: Metrics Fetishism, http://chrishecker.com/Metrics_Fetishism

12. Zamitto, V., Seif EI-Nasr, M., Newton, P.: Exploring Quantitative Methods for Evaluating Sports Games. In: ACM CHI 2010, April 10-15 (2010)

13. Holm, S.: A simple sequentially rejective multiple test procedure. Scandinavian Journal of Statistics 6, 65-70 (1979) 